\title{
The sapphire backscattering monochromator at the Dynamics beamline P01 of PETRA III
}

\author{
$\underline{\text { P. Alexeev }}{ }^{1,3}$, V. Asadchikov ${ }^{4}$, D. Bessas ${ }^{2}$, A. Butashin ${ }^{4}$, A. Deryabin ${ }^{4}$, F.-U. Dill ${ }^{1}$, A. \\ Ehnes $^{1}$, M.Herlitschke ${ }^{1}$, R.P. Hermann ${ }^{3,5,6}$, A. Jafari ${ }^{2,3,6}$, I. Prokhorov ${ }^{4}$, B. Roshin ${ }^{4}$, \\ R.Röhlsberger ${ }^{1}$, K. Schlage ${ }^{1}$, I. Sergeev ${ }^{1}$, A. Siemens ${ }^{1}$, H.-C. Wille ${ }^{1}$ \\ ${ }^{1}$ Deutsches Elektronen-Synchrotron DESY, D-22607 Hamburg, Germany, ${ }^{2}$ European Synchrotron Radiation \\ Facility, F-38043 Grenoble, France, ${ }^{3}$ Jülich Centre for Neutron Science JCNS and Peter Grünberg Institut PGI, \\ JARA-FIT, Forschungszentrum Jülich GmbH, D-52425 Jülich, Germany, ${ }^{4}$ A.V. Shubnikov Institute of \\ Crystallography, Russian Academy of Sciences, R-119333 Moscow, Russia, ${ }^{5}$ Materials Science and Technology \\ Division, Oak Ridge National Laboratory, Oak Ridge, Tennessee 37831, USA, ${ }^{6}$ Faculty of Sciences, \\ University of Liège, B-4000 Liège, Belgium
}

We report on a high resolution sapphire backscattering monochromator installed at the Dynamics beamline P01 of PETRA III. The device enables nuclear resonance scattering experiments on Mössbauer isotopes with transition energies between 20 and $60 \mathrm{keV}$ with sub-meV to meV resolution. In a first performance test with ${ }^{119} \mathrm{Sn}$ nuclear resonance at a Xray energy of $23.88 \mathrm{keV}$ an energy resolution of $1.34 \mathrm{meV}$ was achieved. The device extends the field of nuclear resonance scattering at the PETRA III synchrotron light source to many further isotopes like ${ }^{151} \mathrm{Eu},{ }^{149} \mathrm{Sm},{ }^{161} \mathrm{Dy},{ }^{125} \mathrm{Te}$ and ${ }^{121} \mathrm{Sb}$.

\section{Introduction}

Nuclear resonance scattering (NRS) experiments require for high resolution monochromatization of the X-ray beam. Monochromators with an energy bandwidth of the order of meV are required in order to a) reduce the flux entering the detector in forward direction by reducing energy bandwidth around Mössbauer transition energy and by this to avoid detector overload; b) perform nuclear inelastic scattering (NIS) experiments with an energy resolution sufficient to resolve phonon spectra. Thus, monochromators with an energy resolution of $\Delta E / E<10^{-7}$ where $\mathrm{E}$ is the photon-ray energy and $\Delta E$ is the energy bandwidth, with high spectral reflectivity are required for NRS $[1,2,3]$. The high spectral reflectivity is especially important at energies above $30 \mathrm{keV}$ as the flux at the $3^{\text {rd }}$ generation synchrotron sources decreases in this energy range.

High-index Bragg reflections of Si crystals are used to deliver narrow, sub-meV to meV energy bandwidths in the conventional approach to the monochromatization . A critical parameter for the high spectral reflectivity is the angular acceptance of the Bragg reflection. The angular acceptance drops with energy proportionally to $1 / E^{2}$ at any Bragg angle, except close to $90^{\circ}$. For an efficient monochromator design, the incident X-ray beam divergence should not be larger than the angular acceptance of the reflection, which is on the order of 0.2-10 $\mu \mathrm{rad}$ [3]. One approach to efficiently use high-index Bragg reflections is the angular collimation of the incident beam by a preceding Bragg reflection from an asymmetrically cut crystal. Thus, conventional monochromators consist of several Si crystals providing collimation, energy resolution, and preservation of the direction of the incoming x-ray beam (in-line monochromator). However, these monochromators often become inefficient for energies above $30 \mathrm{keV}$ due to a decrease of the reflectivity, geometrical reasons (extremely 
asymmetrical crystal cuts result in large crystal sizes) and the increasing demands on a precision of the mechanical rotation stages when the Bragg reflection width becomes less than $100 \mathrm{nrad}$. The constraints mentioned above led to the development of high resolution monochromators in backscattering geometry, because at Bragg angles very close to $90^{\circ}$ the angular acceptance is of the order of a few mrad at 20-50 keV and decreases with energy proportionally to $1 / E$. Therefore, a backscattering monochromator consisting of one single crystal can easily cover the incoming beam divergence. Moreover it consist of less moving parts and is comparably easy in operation. The change of the reflected X-ray energy is achieved by changing the temperature of the crystal, i.e. tuning the distance between crystal lattice planes, while the Bragg angle is kept constant. This way of operation requires an extremely precise temperature control over a wide temperature range.

The absolute value of the X-ray energy in NRS experiments is given by the energy of the nuclear transition, which is an isotope specific physical property. Therefore, Bragg reflections near to $90^{\circ}$ covering a specific energy must be reachable in the monochromator crystal at a practical reasonable temperature between about 100 and $400 \mathrm{~K}$, where reflectivity is reasonably large. Unfortunately, silicon due to its cubic symmetry exhibit only a low density of appropriate reflections in energy and practically does not cover most of the desired nuclear energy levels. Furthermore, near $90^{\circ}$ Bragg angle multiple-beam reflections start to interfere with the main reflection reducing its reflectivity. The use of crystals with lower symmetry was suggested in order to avoid this effect [3]. Particularly, high quality sapphire crystals with rhombohedral symmetry became interesting candidates for application in NRS experiments [4]. We report on a sapphire high-resolution backscattering monochromator (BS HRM) at the Dynamics beamline P01. Its performance benefits from a large distance of $21 \mathrm{~m}$ between sample and monochromator at this beamline, which enables a relatively large separation of $15 \mathrm{~mm}$ or more between the direct and the reflected beam at the sample position without compromising the backscattering Bragg condition.

\section{Experimental part}

The most challenging part of the sapphire backscattering monochromator is the temperature control. The smallest measured energy change has to be compared with the energy resolution of the monochromator. Taking into account the characteristic lattice thermal expansion of the sapphire crystal one obtains that the temperature should be controlled with accuracy down to the $1 \mathrm{mK}$. Also temperature changes (e.g for energy scans) must have the same precision in step size.

Thus, the temperature measurement system should be able to acquire smallest temperature changes and the temperature controller should be able to stabilize the temperature around a set point as fast as possible. Moreover, the setup of the monochromator should provide a low vibration level for beam stability.

The constructed at P01 beamline sapphire backscattering monochromator is shown in the Fig.1. The main element is a two-stage flow cryostat. Cold nitrogen gas is used as a coolant, however, any gas can be used which is not reactive to copper, nickel, stainless steel and NBR resin. The cold nitrogen is produced by evaporation of liquid nitrogen from a Dewar and 
supplied to the cryostat via a gas transfer line. The gas temperature is stabilized by preheating the gas transfer line with an accuracy of 200-500 mK.

The cryostat operates at $\sim 1$ bar overpressure, having atmospheric pressure on the outlet. This assures additional temperature stability. The cold gas circulates in the spiral channels of the larger primary and smaller secondary copper heat exchanger and the gas temperature stabilizes down to sub-mK deviations around the set point.

The heat exchanger chamber including the crystal holder is thermally insulated from the outer cryostat wall to avoid thermal transport and resulting cooling power loss. After passing the heat exchangers the tempered cold gas flows inside the crystal enclosure and regulates the temperature of the crystal. The gas is then released at the outlet. The rotameter on the outlet controls the flow and provides better temperature control of the crystal. The typical flow rate is 200-500 liters of nitrogen gas per hour during operation between 150 and $400 \mathrm{~K}$ with 1 bar overpressure.

The backscattering cryostat hosting the sapphire crystal [5] is installed $89 \mathrm{~m}$ downstream of the undulator source and reflects the beam towards the sample that is located $21 \mathrm{~m}$ further upstream. In our first test we used a disk-shaped sapphire crystal with $18 \mathrm{~mm}$ diameter and 5 mm thickness; the surface of the crystal was mechanically polished. The crystal was grown by modified heat-exchange method.

The control of the gas temperature is performed by a platinum PT1000 resistor. A constantan resistive heater is driven by a power supply and connected to a PID-controller [6]. The crystal temperature is measured by a second PT1000 resistor attached to the crystal. The resistor is attached to the crystal mechanically, with a thin layer of silver paste in between the crystal and the resistor. A Keithley 3706 digital multimeter with 8 channels is used for reading of the resistance values. This device reduces noise by a factor of 2 and requires 10 times smaller test currents for measuring $1 \mathrm{kOhm}$ resistances compared to the frequently used $100 \mathrm{Ohm}$ resistances. Thus, the PT1000 resistors with $1088 \mathrm{kOhm}$ resistance at room temperature have been chosen for use in this cryostat.

The monochromator has been tested for temperature stability without heat load from the impinging beam in order to evaluate precision of the temperature measurements, mainly the dependence on the temperature reading rate, at a crystal temperature of $275 \mathrm{~K}$. The temperature point $275 \mathrm{~K}$ was chosen because at this temperature the copper heat exchangers of the cryostat have a relatively high heat capacity which provides a challenge for the reactivity of the temperature control. Moreover, this temperature point corresponds to the energy of $46.5 \mathrm{keV}$ photons reflected by (2 $9 \overline{11}$ 92) sapphire back-reflection, the energy of the Mössbauer transition in ${ }^{183} \mathrm{~W}$ isotope.

The device has also been tested with the heat load provided by $23.88 \mathrm{keV}$ x-ray beam. We used root mean square (r.m.s.) deviation from the mean as a figure of merit to evaluate the sensitivity of temperature measurements. The precision of the temperature control has been studied by r.m.s. of residual sum of squares of the linear approximation of data. 


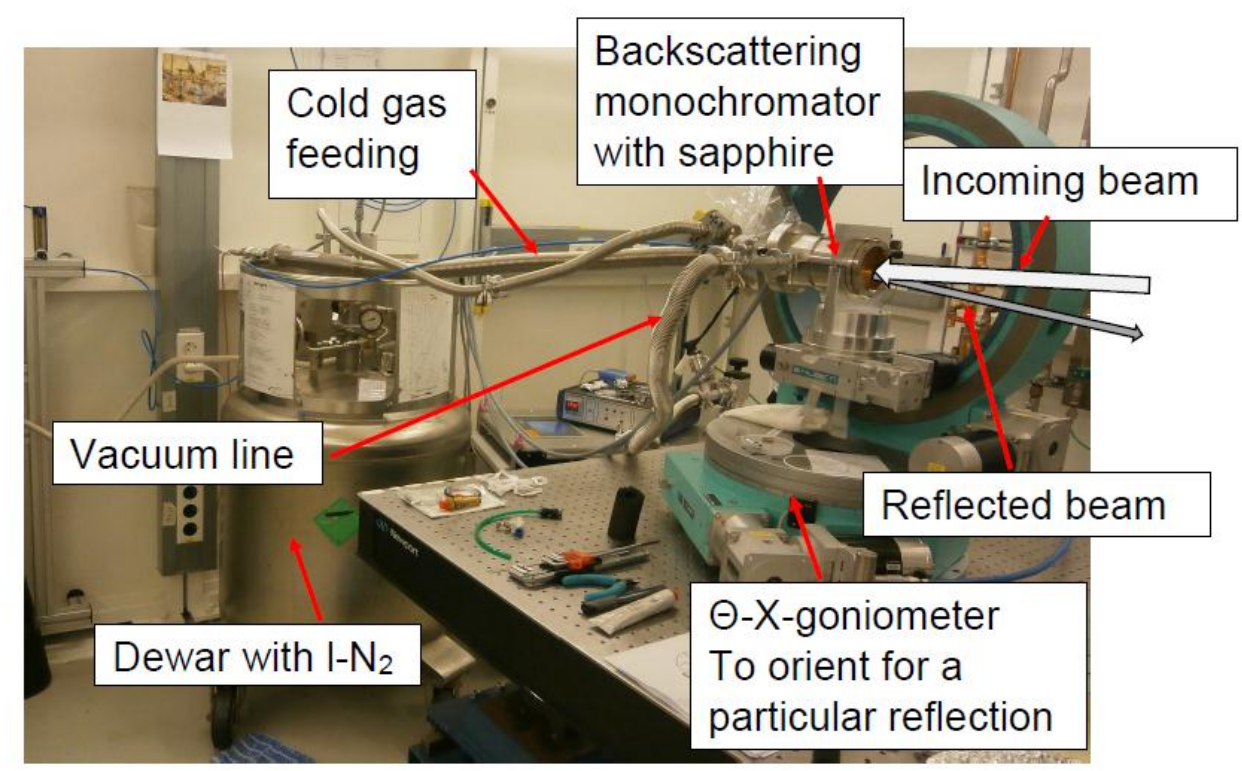

(a)

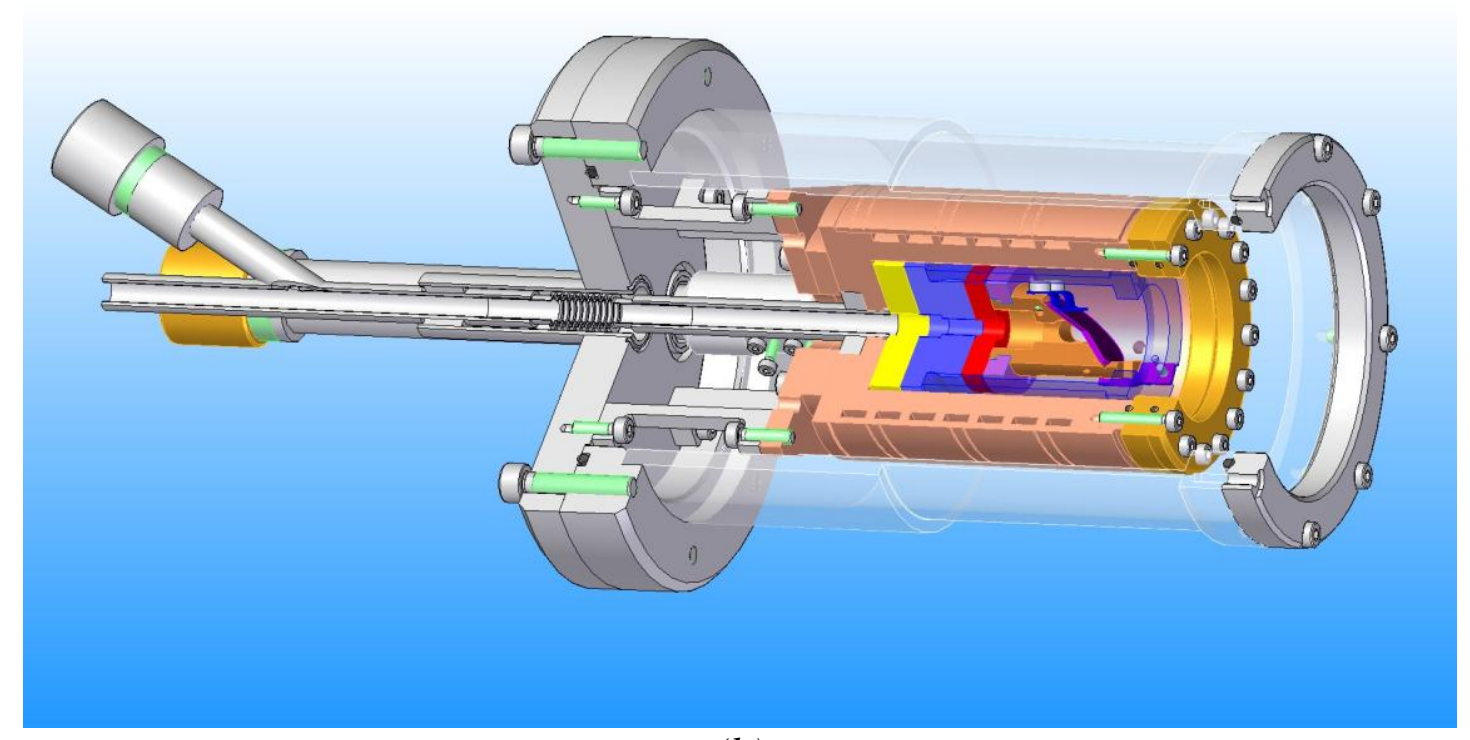

(b)

Fig. 1: Setup (a) and cross-section (b) of the backscattering monochromator.

The sensitivity of the temperature measurements depends on the integration time of the voltage reading by Keithley multimeter. Additionally, the sensitivity depends on the number of channels that are read sequentially. The sensitivity dependence on the integration time is shown in Fig. 2. As follows from this figure, the sensitivity of temperature measurements can be improved by a factor of 10 with an optimal integration time and proper grounding of the measurement circuit, leading to the accuracy of 0.1-0.2 $\mathrm{mK}$. For the purpose of grounding the current probes of the first and fourth channels of the digital multimeter are attached to the earth ground electrode in the rack containing the device.

The results of the temperature control tests are shown in Fig. 3. An average of r.m.s. deviations of $0.181 \mathrm{mK}$ relative to the set point of $275 \mathrm{~K}$ was achieved during each temperature stabilization. 8 temperature stabilization runs each continued for 1 hour in order 
to reveal the repeatability of the control. The achieved precision of $0.181 \mathrm{mK}$ corresponds to an energy drift of the monochromator by $46.6 \mu \mathrm{eV}$ during 1 hour at an energy of $46.5 \mathrm{keV}$. The temperature scans around the temperature $275 \mathrm{~K}$ have been carried out with the temperature ramp $230 \mathrm{mK} / \mathrm{min}$ during $30 \mathrm{~min}$ (Fig 4).

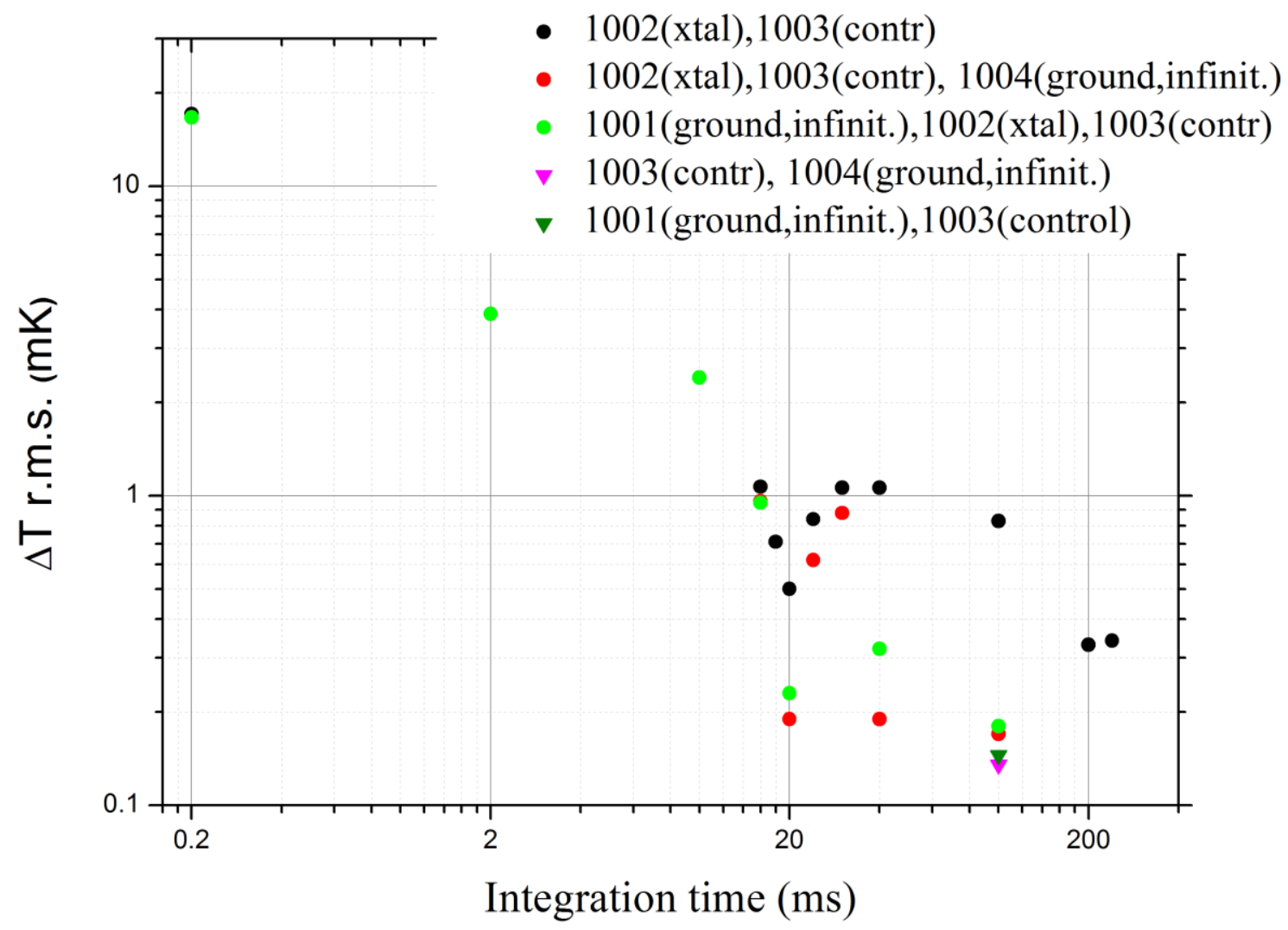

Fig. 2: Sensitivity of resistivity measurements on PT1000 at room temperature for different reading rates and number of channels. Current probe of the channels 1001 and 1004 are grounded, the circuit is opened. Channels 1002 and 1003 are used to measure resistivities of platinum resistances. 


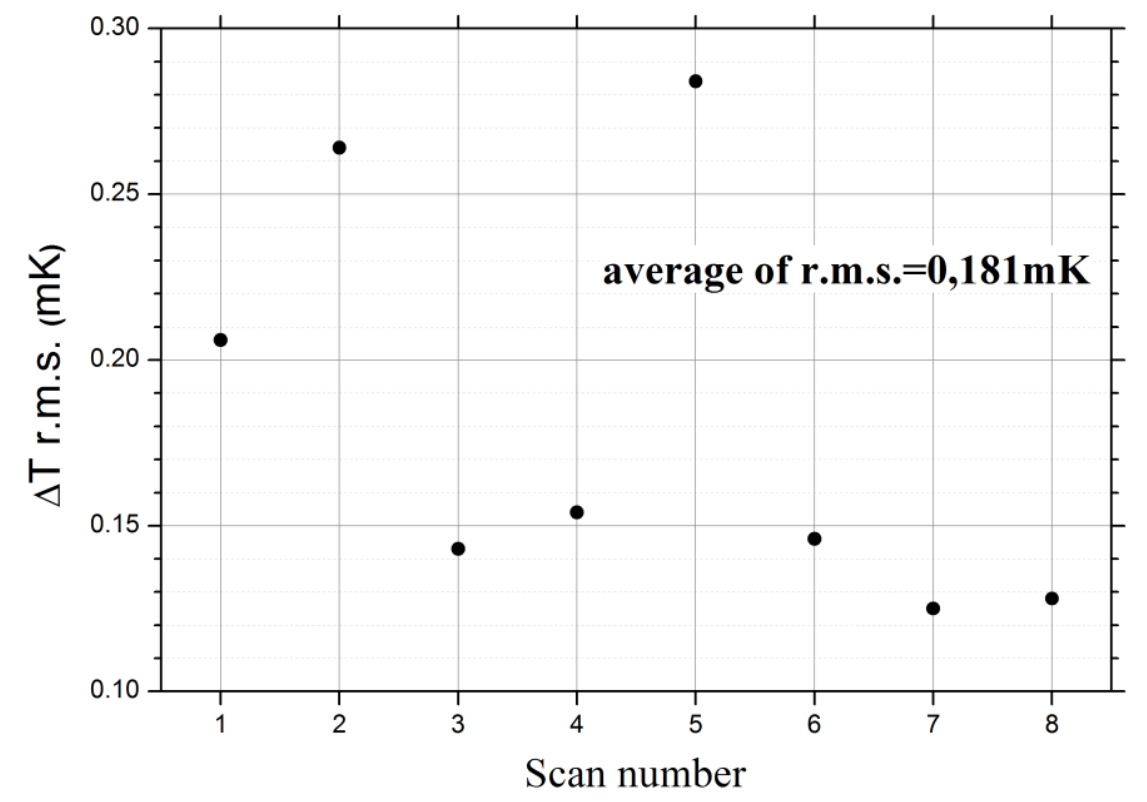

Fig. 3:Temperature stability during temperature control at $275 \mathrm{~K}$. The duration of each scan is 1 hour.

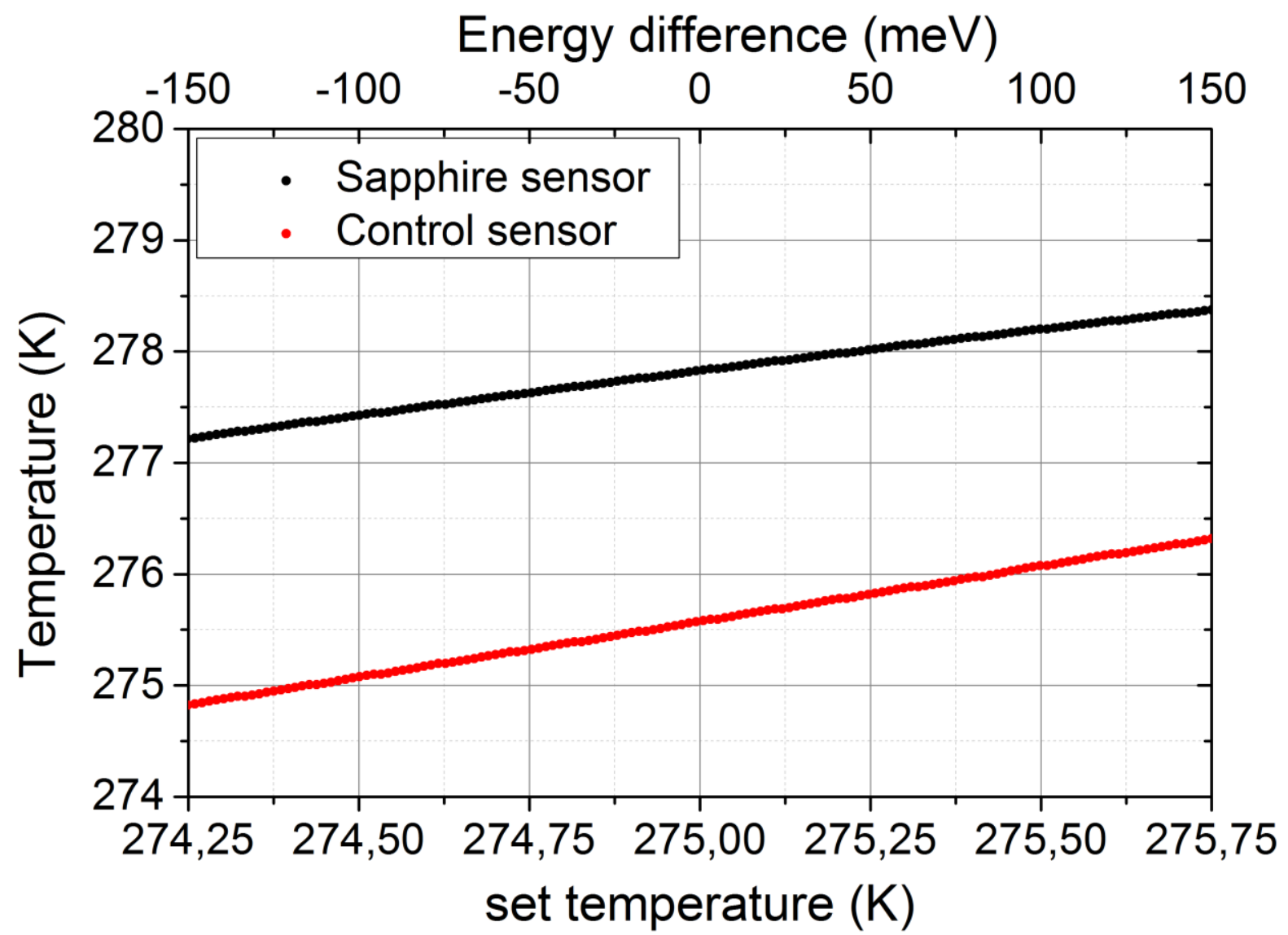

Fig. 4: Temperature control at $275 \mathrm{~K}$ with a fast, $230 \mathrm{mK} / \mathrm{min}$, temperature change. The deviation from linearity is $9.8 \mathrm{mK}$ (r.m.s.) in the temperature range $1.5 \mathrm{~K}$ 


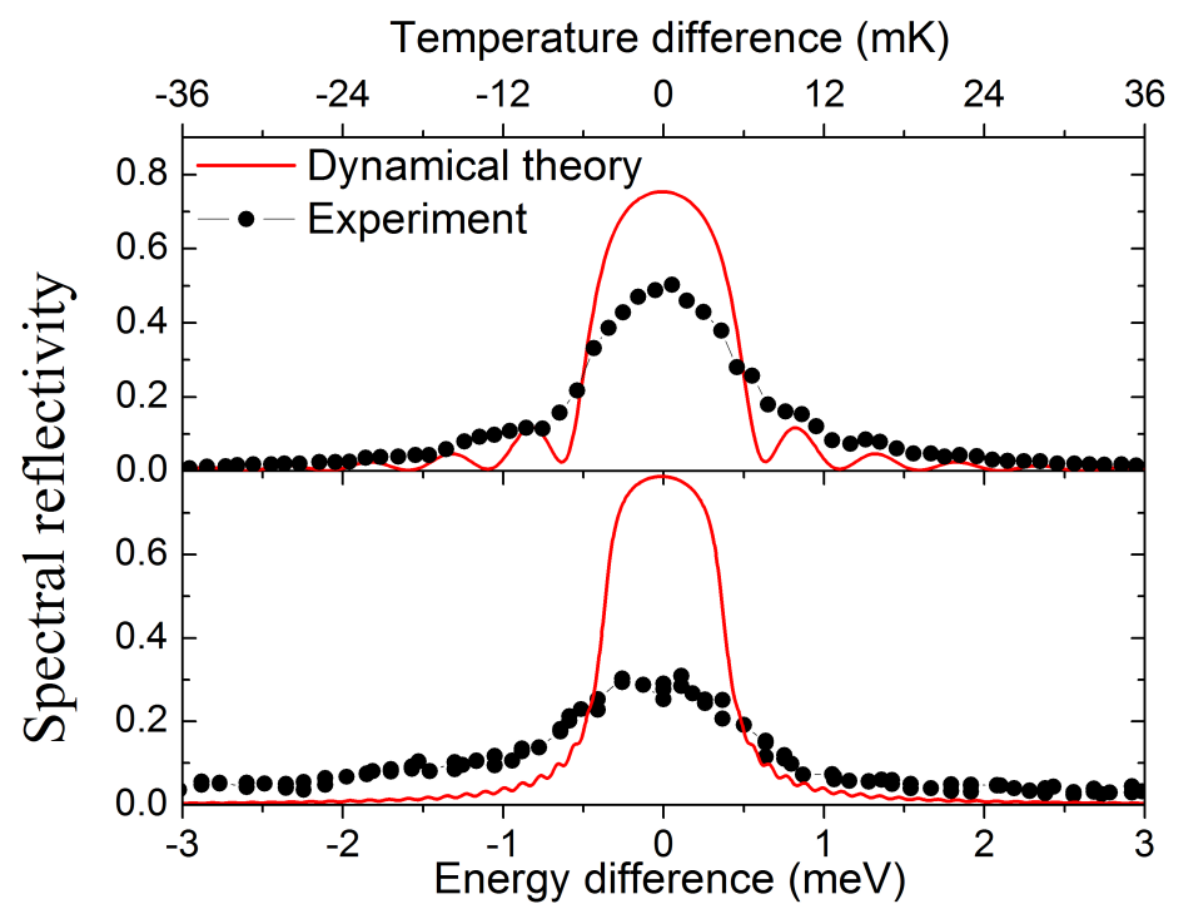

Fig. 5: Spectral reflectivities of $1 \mathrm{~mm}$ thick and 5mm thick sapphire crystals measured around ${ }^{119} \mathrm{Sn}$ resonance energy of $23.88 \mathrm{keV}$ (Energy difference 0 ). Red lines: values calculated by the dynamical theory of $x$-ray diffraction.

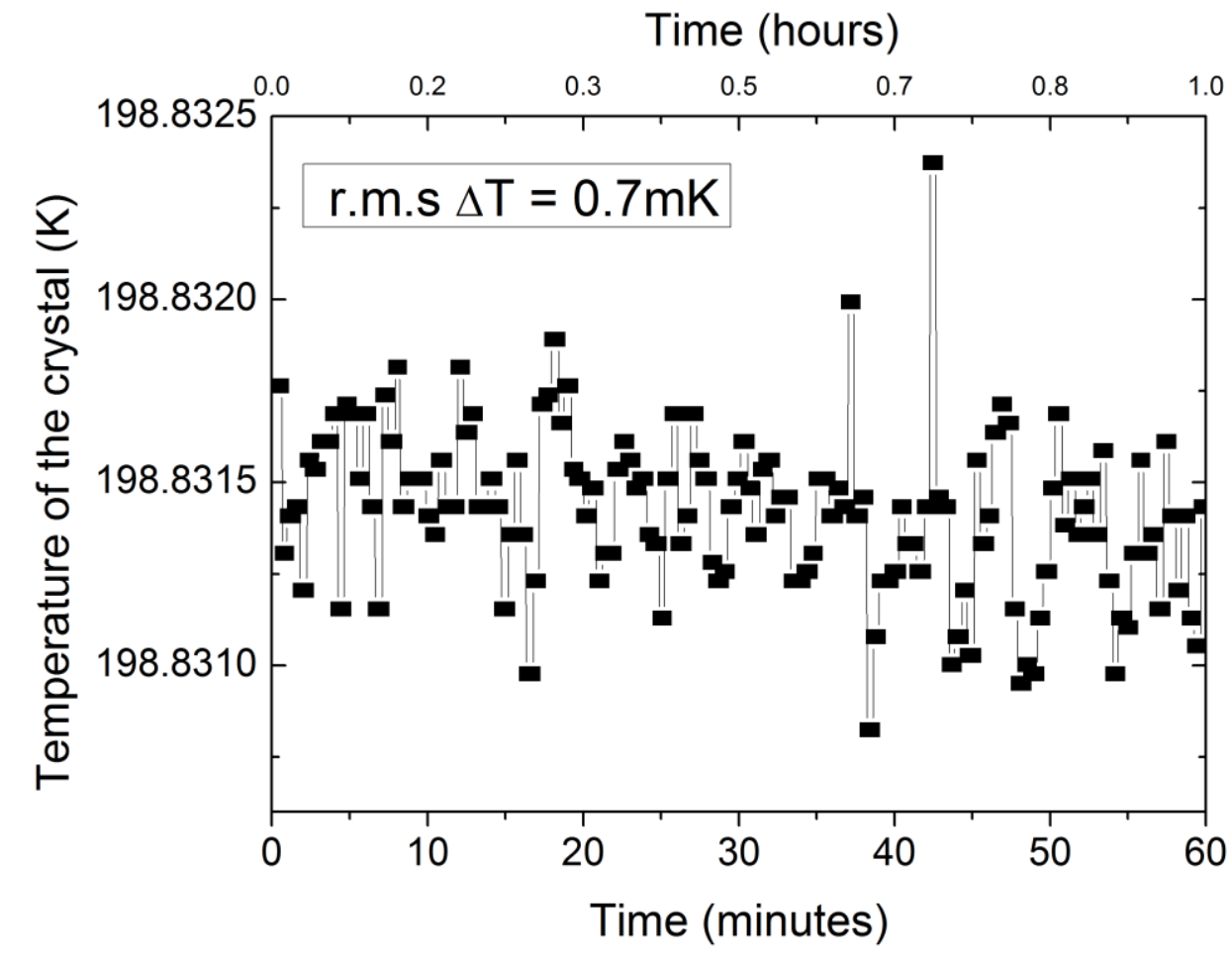

Fig. 6: Measured temperature stability during temperature control with heat load by 23.88 keV $x$-ray beam. The r.m.s. value is $0.7 \mathrm{mK}$ during 1 hour 
The PID temperature controller was optimized by the Ziegler-Nichols method [7]. We noticed that the use of any differential coefficient reduces the stability of the temperature control, thus, only proportional and integral coefficients have been applied to control the temperature.

The test of the backscattering monochromator has been performed with X-ray beam at the energy of $23.88 \mathrm{keV}$ corresponding to the Mossbauer transition of ${ }^{119} \mathrm{Sn}$. The measurements were performed at the P01 beamline, PETRAIII. The synchrotron ring was working at the 40bunch mode in the top-up regime with $95 \mathrm{~mA}$ electron current. The studied sample was $\beta$ ${ }^{119} \mathrm{Sn}$ metal foil enriched in ${ }^{119} \mathrm{Sn}$ isotope to $>95 \%$. The ${ }^{119} \mathrm{Sn}$ resonance was found with the (4 $4 \overline{8} 45$ ) back-reflection of sapphire at a temperature of 199.0(1) $\mathrm{K}$ which corresponds to the energy of $23.8791(5) \mathrm{keV}$ in agreement with [4]. The sapphire quality was inspected by mapping its surface with the instrumental function measured using nuclear forward scattering. A best position on the crystal was found which provides the energy bandwidth of $1.3 \mathrm{meV}$ (FWHM) as shown in Fig.5. Temperature stability at the sapphire crystal was 0.7 $\mathrm{mK}$ (r.m.s.) (fig. 6) which corresponds to the energy broadening of the bandwidth by 0.06 meV which is much smaller than the measured bandwidth. Thus, for this Mossbauer transition the achieved precision of the temperature control is sufficient for the efficient use of the monochromator.

The X-ray flux upstream and downstream of the BSM was measured by a $300 \mu \mathrm{m}$ silicon PIN diode and $100 \mu \mathrm{m}$ thickness APD, respectively. The flux incident on the BS HRM was $8.1 * 10^{12} \mathrm{ph} / \mathrm{s}$ at $23.8793 \mathrm{keV}$. The measured photon flux after the BS HRM was $1.6^{*} 10^{9} \mathrm{ph} / \mathrm{s}$ at this energy. This corresponds to a sapphire spectral reflectivity of $26.6 \%$, or $3.1 * 10^{4}$ photons per natural linewidth $\Gamma_{0}$ of the ${ }^{119} \mathrm{Sn}$ resonance.

At $37.1292(5) \mathrm{keV}$, the flux of the beam incident on the BS HRM was $1.7^{*} 10^{12} \mathrm{ph} / \mathrm{s}$. This value agrees well with the expected value of the flux of $1.2 * 10^{12} \mathrm{ph} / \mathrm{s}$. The sapphire backreflection (8 $16 \overline{24} 40)$ was used for this energy. The monochromatic beam flux after the BS HRM was $8.1 * 10^{7} \mathrm{ph} / \mathrm{s}$. Due to spurious bunches in the PETRA ring we were not able to observe the ${ }^{121} \mathrm{Sb}$ resonance and could not measure the instrumental function of the BS HRM at this energy. To give an estimation, we assume that the instrumental function was the same as for $23.8793 \mathrm{keV}$. Thus, the spectral reflectivity after the BS HRM can be estimated as 11.9 $\%$ at $37.1292 \mathrm{keV}$, or $7900 \mathrm{ph} / \mathrm{s}$ per natural linewidth $\Gamma_{0}$ of the ${ }^{121} \mathrm{Sb}$ resonance.

\section{Discussion}

The reading rate of the temperature significantly influences the precision of temperature measurements. With decreasing the reading rate from $5000 \mathrm{~s}^{-1}$ to $50 \mathrm{~s}^{-1}$ the sensitivity of the temperature measurements increases by almost 2 orders of magnitude. This increase is related to the averaging of the thermal effects on the contacts, Josephson current, and thermal drift on the platinum resistor. However, it is interesting to note that a further decrease of the reading rate from $50 \mathrm{~s}^{-1}$ to $30 \mathrm{~s}^{-1}$ decreases the sensitivity by a factor 5 , which is not yet understood. The highest sensitivity of the temperature measurements from 2 channels, $0.33 \mathrm{mK}$, was reached with minimal reading rate $50 \mathrm{~s}^{-1}$.

Using three measurement channels we observed that the grounding of one of 8 available channels, while reading from 2 other channels improves temperature sensitivity by almost factor 3, down to $0.181 \mathrm{mK}$. This observation can be related to the removal of parasitic currents by grounding of the measurement circuit.

The tested precision of the temperature control shows an energy drift of the monochromator $46.6 \mu \mathrm{eV}$ during 1 hour at the energy $46.5 \mathrm{keV}$. 
In a typical NIS experiment energy scans are performed over the range $+/-150 \mathrm{meV}$ around the resonance line within a scan time of 30 to 120 minutes. The higher the temperature change over time is, the higher the non-linearity. As an extreme case we tested the temperature control by performing a $300 \mathrm{meV}$ scan within 30 minutes and received a deviation from linearity $9.8 \mathrm{mK}$ (r.m.s.) (fig. 4). This deviation corresponds to the $\sim 0.437$ meV relative energy uncertainty at $46.5 \mathrm{keV}$. This value is still reasonable since sapphires currently used for the backscattering monochromators provide resolutions not better than 0.9 $\mathrm{meV}$. The non-linearity can be strongly reduced by a reduced temperature change rate. The sapphire with $5 \mathrm{~mm}$ thickness utilized herein shows a factor 1.5 less spectral reflectivity at $23.88 \mathrm{keV}$ than that with $1 \mathrm{~mm}$ thickness utilized in Ref. 4 . This fact is related to the lower quality of the $5 \mathrm{~mm}$ thick crystal compared to that of $1 \mathrm{~mm}$ crystal. Also, in contrast to the 5 $\mathrm{mm}$ crystal, the $1 \mathrm{~mm}$ crystal has been studied with a focussed incident beam, thus, probability to find a defect-free region was higher for this crystal. However, according to calculations, a $5 \mathrm{~mm}$ thick sapphire has an almost factor 10 higher reflectivity at energies above $40 \mathrm{keV}$ compared to a $1 \mathrm{~mm}$ thick sapphire. Thus, further improvement of the sapphire crystal quality is of paramount importance.

The values of the photon flux obtained at the energies of ${ }^{119} \mathrm{Sn}$ and ${ }^{121} \mathrm{Sb}$ resonances assure the applicability of the backscattering monochromator for NRS experiments on compounds containing these isotopes and indicates that also many isotopes mentioned in the table 1 will be accessible at the Dynamics Beamline P01, soon.

Table 1: Some isotopes with transition energies $E_{\gamma}$ suitable to sapphire monochromator, lifetimes, sapphire backscattering reflections and sapphire temperatures that match the transition energies [1, 4].

\begin{tabular}{|l|l|l|c|l|}
\hline Isotope & $\mathrm{E}_{\gamma}, \mathrm{keV}$ & Life time, ns & Sapphire back-reflection & Sapphire temperature, K \\
\hline${ }^{151} \mathrm{Eu}$ & $21.5412(5)$ & 13.99 & $(32 \overline{5} 43)$ & 289 \\
\hline${ }^{149} \mathrm{Sm}$ & $22.5015(5)$ & 10.27 & $(510 \overline{15} 22)$ & 252 \\
\hline${ }^{119} \mathrm{Sn}$ & $23.8793(5)$ & 25.61 & $(44 \overline{8} 45)$ & 193 \\
\hline${ }^{161} \mathrm{Dy}$ & $25.65136(3)$ & 41.26 & $(35 \overline{8} 49)$ & 232 \\
\hline${ }^{125} \mathrm{Te}$ & $35.4920(5)$ & 2.14 & $(91 \overline{10} 68)$ & 220 \\
\hline${ }^{121} \mathrm{Sb}$ & $37.1292(5)$ & 4.99 & $(816 \overline{24} 40)$ & 237 \\
\hline
\end{tabular}

\section{Acknowledgements}

We are grateful to the Helmholtz association of German research centers, project HRJRG402 "Sapphire ultra-optics for synchrotron radiation", and the Russian academy of science for supporting this project. PETRA III and ESRF synchrotron radiation sources are acknowledged for provision of beam time at P01 and ID18, respectively.

We are thankful to the mechanical workshops of the DESY photon science department and Research Center Jülich GmbH, Dr. B. Klobes, J. Herda, I. Schwark and Dr. O. Leupold for the technical support, comments, and fruitful discussions. We are grateful to Dr. T. Nunez for help with the implementation of the software at the P01 beamline. Dr. A. Chumakov and J.-P. Celse are acknowledged for the helpful discussions concerning the crystal holders. RPH 
acknowledges support from the Materials Sciences and Engineering Division, Office of Basic Energy Sciences, U.S. Department of Energy.

\section{References}

[1] R. Röhlsberger, Nuclear Condensed Matter Physics with Synchrotron Radiation: Basic Principles, Methodology and Applications, Springer Tracts in Modern Physics, Vol. 208, Springer-Verlag Berlin Heidelberg (2004)

[2] T. Toellner, Hyperf. Interact. 125, 3 (2000)

[3] Yu. Shvyd'ko, X-ray Optics: High-Energy-Resolution Applications, Springer Series in Optical Sciences, Vol.98, Springer, Berlin (2004)

[4] I. Sergueev, H.-C. Wille, R. P. Hermann, D. Bessas, Yu. V. Shvyd'ko, M. Zając, and R.Rüffer, J. Synch. Rad. 18, 802 (2011).

[5] P. van der Linden, H.-C. Wille, and Yu. V. Shvydko, AIP Conf. Proc. 879, 915 (2007).

[6] M. Lucht, PhD thesis, University of Hamburg (1998).

[7] J.G. Ziegler and N.B. Nichols, Transactions of the ASME 64, 759 (1942) 